\title{
BMJ Open Spatial-temporal dynamics and structural determinants of child and maternal mortality in a rural, high HIV burdened South African population, 2000-2014: a study protocol
}

\author{
B Tlou, ${ }^{1,2}$ B Sartorius, ${ }^{1,2}$ F Tanser ${ }^{1,2,3}$
}

To cite: Tlou B, Sartorius B, Tanser F. Spatial-temporal dynamics and structural determinants of child and maternal mortality in a rural, high HIV burdened South African population, 20002014: a study protocol. BMJ Open 2016;6:e010013. doi:10.1136/bmjopen-2015010013

- Prepublication history for this paper is available online. To view these files please visit the journal online (http://dx.doi.org/10.1136/ bmjopen-2015-010013).

Received 21 September 2015 Revised 24 March 2016 Accepted 5 April 2016

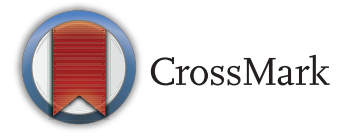

${ }^{1}$ Discipline of Public Health Medicine, School of Nursing and Public Health, University of KwaZulu-Natal, Durban,

South Africa

${ }^{2}$ Africa Centre for Health and Population Studies, University of KwaZulu-Natal, Mtubatuba, South Africa

${ }^{3}$ Centre for the AIDS

Programme of Research in South Africa- CAPRISA, University of KwaZulu-Natal, Congella, South Africa

Correspondence to B Tlou; Tlou@ukzn.ac.za

\section{ABSTRACT}

Introduction: Child (infant and under-5) and maternal mortality rates are key indicators for assessing the health status of populations. South Africa's maternal and child mortality rates are high, and the country mirrors the continental trend of slow progress towards its Millennium Development Goals. Rural areas are often more affected regarding child and maternal mortalities, specifically in areas with a high HIV burden. This study aims to understand the factors affecting child and maternal mortality in the Africa Centre Demographic Surveillance Area (DSA) from 2003 to 2014 towards developing tailored interventions to reduce the deaths in resource poor settings. This will be done by identifying child and maternal mortality 'hotspots' and their associated risk factors.

Methods and analysis: This retrospective study will use data for 2003-2014 from the Africa Centre Demographic Information System (ACDIS) in rural KwaZulu-Natal Province, South Africa. All homesteads in the study area have been mapped to an accuracy of $<2 \mathrm{~m}$, all deaths recorded and the assigned cause of death established using a verbal autopsy interview. Advanced spatial-temporal clustering techniques (both regular (Kulldorff) and irregular (FleXScan)) will be used to identify mortality 'hotspots'. Various advanced statistical modelling approaches will be tested and used to identify significant risk factors for child and maternal mortality. Differences in attributability and risk factors profiles in identified 'hotspots' will be assessed to enable tailored intervention guidance/development. This multicomponent study will enable a refined intervention model to be developed for typical rural populations with a high HIV burden.

Ethics: Ethical approval was received from the Biomedical Research Ethics Committee (BREC) of the University of KwaZulu-Natal (BE 169/15).

\section{BACKGROUND}

Substantial global progress has been made in reducing child deaths since 1990 , specifically in resource poor settings. The number of under-5 deaths worldwide has declined from 12.7 (12.6, 13.0) million in 1990 to 5.9 (5.7, 6.4) million in 2015, amounting to 16000 every day compared with 35000 in $1990 .{ }^{1}$ Sub-Saharan Africa (SSA) has the highest child mortality rates, this being 98 deaths per 1000 live-births for under-5, more than 15 times the average for developed regions. ${ }^{2}$ Child mortality in South Africa has been reduced from 60 per every 1000 in 1990 to 41 per every 1000 in 2015 , with an annual reduction rate of 1.6, with the 2015 target for Millennium Development Goal (MDG) 4 being 20 per every $1000 .{ }^{1}$ It can therefore safely be concluded that this MDG has not been met, although there was a reduction from 1990 to 2015. HIV and AIDS are still the largest cause of the country's fatalities in children younger than 5, accounting for $40 \%$ of deaths. ${ }^{1}$ It has a high prevalence of HIV and large socioeconomic differences within and among provinces. However, the rate of improvement in HIV care in terms of access to antiretroviral treatment since 2005 has been impressive, the fourth fastest globally and second only to Rwanda among African countries. $^{3}$

MDG 4 targeted reducing the global under-5 mortality rate by two-thirds between 1990 and 2015. In order to achieve this on time, the reduction rate would have had to rise to $15.6 \%$ for $2012-2015$, much greater than the $3.9 \%$ achieved from 2005 to $2012 .^{2}$ Globally, the maternal mortality ratio (MMR) fell by nearly $44 \%$ over the past 25 years, from 385 (UI 359 to 427) per 100000 livebirths in 1990 to an estimated $216(80 \%$ uncertainty interval (UI) 206 to 249) in 2015. ${ }^{4}$ The main target of MDG 5 was a three-quarters reduction in the maternal 
mortality ratio (MMR) between 1990 and 2015. However, recent data suggest that there has been little or no progress in South Africa towards achieving this goal, as it has increased from 108/1000 in 1990 to 138 in 2015, due largely to the growing HIV/AIDS pandemic. ${ }^{4}$ Thus, despite progress in the expanding health infrastructure and improving access to health services, further reductions/improvements are required.

Risk factors for child mortality vary globally in developing and developed countries, with over $70 \%$ of all under-5 deaths occurring in African and South-East Asia regions. ${ }^{5}$ In SSA, the major causes of childhood mortality, summarised as disease and malnutrition, are exacerbated by socioeconomic factors, including access to treatment and care. In South Africa, slow progress in ensuring access to HIV treatment is still among the main causes of child mortality. As of 2014, only $32 \%$ of the $\sim 2.6$ million children living with HIV had been clinically diagnosed, and only $32 \%$ of children living with HIV had access to antiretroviral therapy. ${ }^{6}$ Other leading causes of death in under-5 children are pneumonia, preterm birth complications, birth asphyxia, diarrhoea and malaria. In addition, it is important to mention maternal death as a risk factor for child mortality. Children with deceased mothers often suffer from a lack of day-to-day care, isolation, as well as the consequences of the economic costs associated with their mother's death. ${ }^{7}$ A study conducted in South Africa found that children who experienced an early maternal death were at 15 times the risk of dying before the age of 1 month (RR 15.2; 95\% CI 8.3 to 27.9) than those whose mothers survived. $^{8}$

The overwhelming majority of maternal deaths globally arise from the risks attributable to pregnancy and childbirth, as well as from the poor performance of health services. While many countries are making progress, maternal mortality in South Africa (SA) increased significantly from 1990 to 2015 due largely to the HIV/AIDS pandemic. ${ }^{9}$ The main direct causes of maternal death in developing countries include haemorrhage, sepsis, obstructed labour and hypertensive disorders, ${ }^{10}$ and is influenced by the behaviour of families and communities, social status, education, income, nutritional status, age, parity and the availability of health services.

The Mosley-Chen and Meade models ${ }^{11}{ }^{12}$ will provide the conceptual framework for this study to interrelate child and maternal mortality determinants in the study area. The basis of the Mosley-Chen model is that the underlying socioeconomic status manifests itself in proximate determinants, with the values for these variables influencing the risk of disease, which in turn links the probability of death. The proposed framework will integrate ecological, social and biological risk factors for child and maternal mortality. Structural equation modelling techniques will be used for testing and estimating causal relations using a combination of statistical data and qualitative causal assumptions. Figure 1 summarises a proposed conceptual framework for modelling the risk factors of child and maternal mortality in relation to the Mosley-Chen and Meade models. ${ }^{11} 12$

\section{Motivation for focusing on rural areas and population-based longitudinal cohort (demographic surveillance system) change}

Studies elsewhere have indicated that high child mortality rates are experienced in rural areas of South Africa, with research in a rural setting where HIV infection is endemic indicating that children are still at high risk of dying. ${ }^{13}$ This would provide an in-depth analysis to determine which sociodemographic characteristics tend to place children and mothers at higher risk of death in a high HIV burdened rural South African setting.

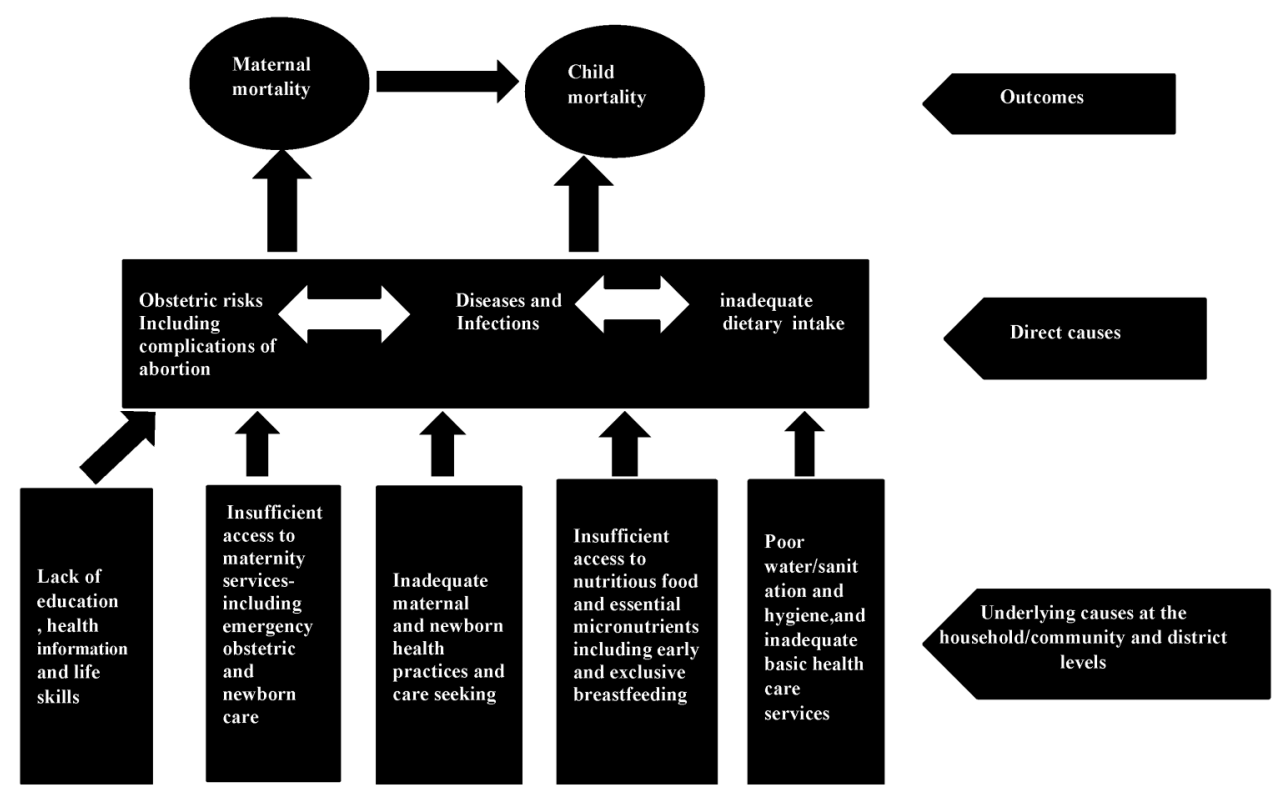

Figure 1 Theoretical conceptual framework underpinning the study. ${ }^{11} 12$ 
This will help to inform primary healthcare practices and facilitate targeting community health worker efforts, specifically in well-defined catchment areas.

Understanding the impact of location on health is a key element of epidemiological investigation, and numerous spatial-temporal methods can be employed to analyse spatial health-related data. Interest in 'place' has experienced a resurgence, facilitated by improved accessibility of computer-based geographic information systems (GIS). This study will use advanced spatiotemporal techniques to identify high-risk areas for child and maternal mortality, as this will guide health planners and policymakers in the effective use of scarce resources to target intervention programmes. Targeting high-risk areas is important for prevention and treatment efforts to reach high-risk populations and hot spots. Methodologically identifying best choice modelling approaches for large georeferenced longitudinal data sets is important in identifying risk actors in terms of attributability to assess relative impact for further policy guidance.

The Aim of the study is to understand the factors affecting child and maternal mortality in the DSA from 2003 to 2014 towards developing tailored interventions to reduce the deaths in resource poor settings.

The study objectives are to:

1. Identify space-time clustering (hotspots) of child mortality

2. Identify space-time clustering (hotspots) of maternal mortality

3. Identify the 'best model' approach (logistic, discrete time, time-to event, Bayesian, SEM) to predict child or maternal mortality
4. Identify high impact risk factors for child and maternal mortality using the 'best model'

5. Explore the impact of timing of mother's death on child survival after adjustment for potential risk factors identified in objective 4 .

\section{Methodology}

Study design

This will be a retrospective analysis of data from an ongoing longitudinal population-based cohort that enables long-term follow-up, characterisation of cause of death patterns in absence of quality/routine vital registration data, and for causal inferences to be made when assessing contextually relevant risk factors. This type of longitudinal data enables the identification of long-term temporal trend changes, and hence the impact of previous health policy shifts intervention programme changes.

\section{Setting}

The Africa Centre Demographic Surveillance Area is located in the rural UMkhanyakude District of KwaZulu-Natal (KZN) Province, South Africa. The surveillance area (figure 2) is located near the market town of Mtubatuba and covers an area of $438 \mathrm{~km}^{2}$, with a population of $\sim 85000$ people who are members of roughly11000 households. ${ }^{14}$ The Africa Centre Demographic Information System (ACDIS) contains data about residents in the area as well as the co-ordinates of all homes in the area, which has enabled the data to be spatially mapped in the Centre's GIS. Unemployment in the area is high, with many families
Figure 2 Location of the Africa Centre demographic surveillance site in KwaZulu-Natal, South Africa. $^{14}$

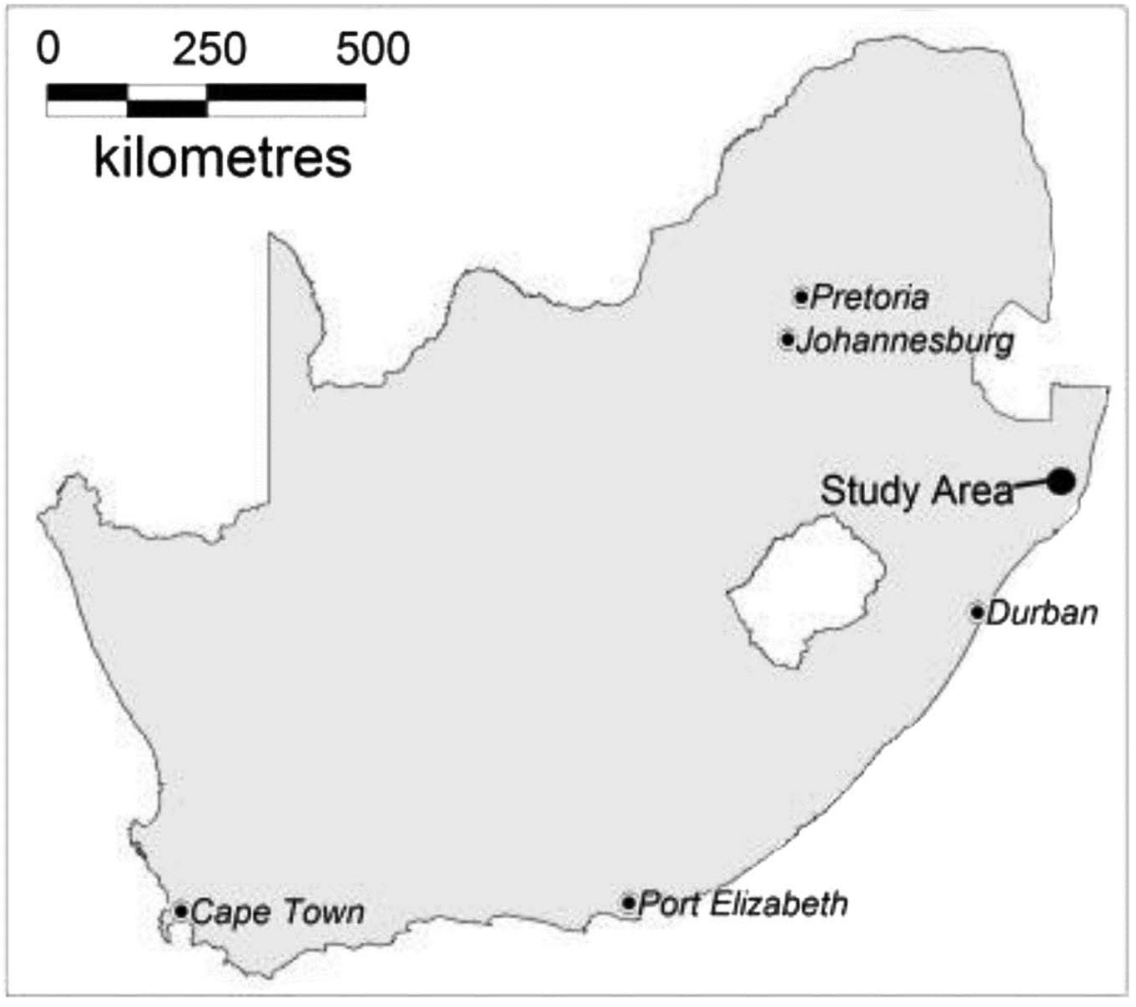


relying on social grants and migrant wages to pay for goods and services. Most of the area is not electrified, roads are seldom tarred and the homes range from traditional to modern structures.

\section{Study participants}

As a retrospective chart review, the participants for this study will be the resident children $(<5$ years $)$ and their mothers who lived within the surveillance area over the study period of 2003-2014. Regarding the children, they will be included if they died of any cause related to or aggravated by pregnancy or its management, excluding accidental or incidental causes of death, while the mothers will be included if they die within 42 days of delivery) ${ }^{15}$ and mother mortality (death while the child aged $<5$ years).

\section{Conceptual framework}

This study will adopt the Mosley-Chen and Meade models to identify the risk factors affecting child and maternal mortality based on three levels: community, household and individual. A structural version of the conceptual framework is presented in figure 1, but for the purposes of this study only selected variables will be included. The key variables used in this study will be: community (distance to local clinics and inadequate healthcare services), household (poor water services, malnutrition, sanitation and hygiene, education and socioeconomic status) and individual (age, gender, HIV status, complications of abortion, diseases and infections).

\section{Study data}

All the data will be obtained from the ACDIS, which is routinely collected over two cycles, one for household level data and the other for individuals. ${ }^{14}$ For the former, a set of questionnaires is routinely administered every 6 months to a key informant in each household. Individual level data are collected annually by interviewing the most senior eligible family member, and include the HIV serosurvey.

To meet objectives 1 and 2, data on childhood and maternal mortality will be extracted from the ACDIS, and will include age, cause of death, as well as coordinates of the affected homesteads. To meet objective 4, the risk factors listed below will be extracted from the ACDIS, in line with the key variables indicated in the Mosley-Chen and Meade models that will be used in this study. To meet objective 5 , data relating to the potential risk factors identified in objective 4 will be extracted from the ACDIS to enable any relationship between maternal and child deaths to be established.

Insufficient access to maternity services-poor environmental health facilities; and inadequate and limited access to basic healthcare services.

Malnutrition-lack of proper nutrition, caused by not having enough to eat, not eating enough of the right things.
Sanitation-conditions relating to public health, especially the provision of clean drinking water and adequate sewage disposal.

Disease - a condition of the living animal or body or of one of its parts that impairs normal functioning and is typically manifested by distinguishing signs and symptoms.

Infection-a disease that is caused by bacteria or a virus.

\section{Classification of cause of death}

All deaths reported in the household surveillance survey are followed up using a verbal autopsy (VA), that is, an interview using a validated structured questionnaire with the closest caregiver of the deceased. This study only included residents (not migrants) for causes of mortality as their episodes are taken into account while computing person time and thus mortality rates (MRs). VA is an epidemiological tool that is used to establish the cause of death in settings where hospital-based records are lacking or inadequate. During the period of this study, a VA interview was conducted on average 9 months after a death was reported. In interviews conducted by a trained nurse, informants were asked to narrate the course of the illness or events leading to death. Two experienced clinicians independently assigned the underlying cause of death based on the information collected in the VA and their clinical judgement using the 10th version of the International Classification of Diseases (ICD-10) coding system. Following the Global Burden of Disease classification, the causes were divided into three broad categories: (1) communicable, maternal, perinatal and nutritional conditions; (2) non-communicable diseases; and (3) injuries. ${ }^{16}$

\section{Statistical analyses}

The data will be processed and analysed using Stata V.13.0 to meet the five study objectives (S. T. Support, 'Citing Stata software, documentation, and FAQs,' 2015). For objectives 1 and 2, quadrat analysis, kernel estimation, nearest neighbour distances and $\mathrm{k}$ functions for point pattern analysis and spatial dependence will be used. ${ }^{17}$ This will help to determine if there is a tendency of events to exhibit a systematic pattern over an area as opposed to being randomly distributed. The regular shaped Kulldorf spatial-temporal and irregular shaped Flexible scan statistics will be used for cluster detection. These will be implemented in SaTScan and FleXScan software packages, respectively ('SatScan.' http://www. satscan.org/related_software.html (accessed 23 Jul 2015); K Takahashi, T Yokohama, T Tango. Flexiscan: Software for the flexible scan statistics 2005). https://sites.google. com/site/flexscansoftware/ (accessed 31 Jul 2015). For objective 3 , a generalised structural equation modelling (GSEM) approach for individual level data using logistic regression will be used to establish the direct and indirect risk factors for child and maternal mortality. A structural version of the proposed conceptual framework to be 
Figure 3 A structural version of the proposed conceptual framework to be confirmed.

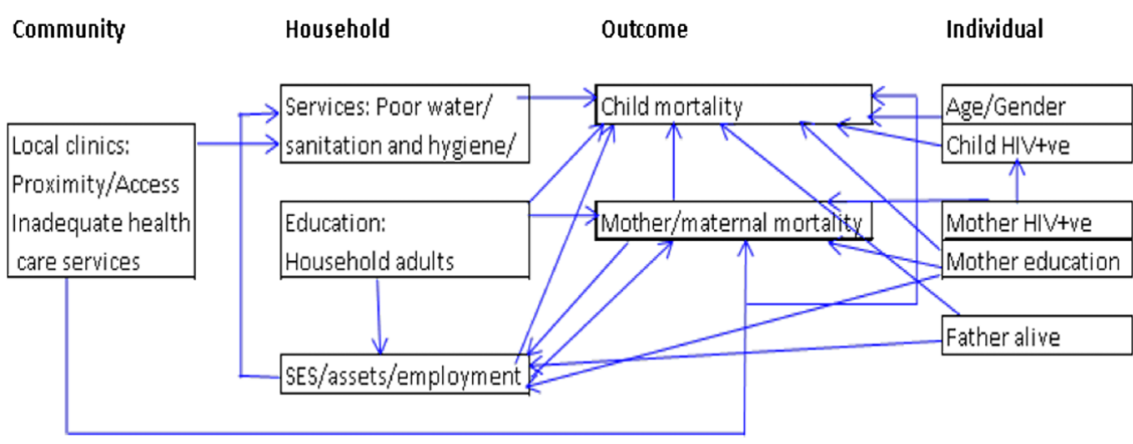

confirmed is presented in figure 3. Community, household and individual variables will be treated as explanatory (exogenous) variables while child and maternal mortality will be used as response variables. Maternal mortality will be used as both explanatory and outcome (endogenous) variables. The relationships between continuous predictive variables will be explored using linear regression techniques. Model fit will be assessed using the $\chi^{2}$ goodness of fit or numerous indicators of model fit such as the normed fit index, the comparative fit index (CFI) or the root mean square error of approximation. The parameter estimation will be done using maximum likelihood in STATAV.13.

A preliminary bivariate Cox survival analysis will first be conducted to explore the relationship between child and maternal mortality with each risk factor. Covariates significant at the $10 \%$ level will then be incorporated into the multivariable model. A multivariable Bayesian geospatial Cox frailty model will be used to determine factors associated with the risk of child mortality while adjusting for inherent spatial correlation. Assuming that $\mathrm{T}_{\mathrm{ij}}$ is the observed number of months lived or censoring time for the $\mathrm{j}^{\text {th }}$ child in areai, the baseline hazard function at time $\mathrm{T}=\mathrm{t}$ is given by

$$
\mathrm{h}\left(\mathrm{t} \mid \beta, \mathrm{v}_{\mathrm{ij}}\right)=\mathrm{h}_{0}(\mathrm{t}) \exp \left(\beta \mathrm{v}_{\mathrm{ij}}\right)
$$

where $h_{0}(t)$ is the baseline hazard at time $t$, and $\beta$ are a vector of regression coefficients for the fixed and time-invariant variables $\left(\mathrm{v}_{\mathrm{ij}}\right)$. Coefficients will be exponentiated to represent the HRs, that is, the ratio of instantaneous risks which is assumed to be constant over time.

Since individuals reside in households, and spatial correlation in outcomes becomes increasingly important as household proximity decreases, a spatially structured household random frailty term, $\psi_{\mathrm{i}}$, will be introduced to augment the Cox model as well as an unstructured individual level frailty term, $\mu_{\mathrm{i}}$, to capture any unobserved heterogeneity:

$$
\mathrm{h}\left(\mathrm{t} \mid \beta, \mathrm{v}_{\mathrm{ij}}, \psi_{\mathrm{i}}\right)=\mathrm{h}_{0}(\mathrm{t}) \exp \left(\beta \mathrm{v}_{\mathrm{ij}}+\psi_{\mathrm{i}}+\mathrm{u}_{\mathrm{i}}\right)
$$

where $\beta$ is assumed to be $\sim \operatorname{Normal}(0,0.1)$ and $\psi_{\mathrm{i}} \psi$ follows a multivariate normal distribution, $\psi_{\mathrm{i}} \sim \operatorname{MVN}(0, \Sigma)$ with variance-covariance matrix $\Sigma$ expressed as a parametric function of distance between households. The simplest form (isotropic) will be employed, the assumption being that spatial correlation between any two locations is a function of Euclidean (straight-line) distance. The individual level unstructured frailty will follow an independent normal distribution $u_{i} \sim N$ $\left(0, \sigma^{2} u\right)$. Non-informative inverse gamma priors will be adopted for the variance parameters.

MCMC simulation will be used to fit the Bayesian models. ${ }^{18}$ Convergence will be assessed by visual inspection of the parameter series plots, as well as with Gelman-Rubin statistics. ${ }^{19}$

Deviance Information Criterion (DIC) will be used to assess the fit of the Bayesian models, that is, the one providing the smallest DIC will be assumed to be a better fitting model. ${ }^{20}$

For discrete-time events analysis, the discrete-time hazard function will be modelled using the equation below:

$$
\mathrm{p}_{\mathrm{ti}}=\operatorname{Pr}\left(\gamma_{\mathrm{ti}}=1 \mid \gamma_{\mathrm{t}-1, \mathrm{i}=0}\right)
$$

$\mathrm{p}_{\mathrm{ti}}$ is a discrete-time approximation to the continuoustime hazard function $h_{i}(t)$.In the multinomial logit model, the log-odds of each response will be assumed to follow a linear model

$$
\eta_{i j}=\log \frac{\pi_{i j}}{\pi_{i j}}=\alpha_{j}+\chi_{i}^{\prime} \beta_{j}
$$

where $\alpha_{\mathrm{j}}$ is a constant and $\beta_{\mathrm{j}}$ is a vector of regression coefficients for $\mathrm{j}=1,2, \ldots, \mathrm{J}-1$. Bayesian geostatistical models using the Win Bugs software will be used in this study in order to address the independence problems, which traditional statistical methods may not, and the comparison of clusters to non-clusters. ${ }^{21}$

Population attributable fractions (PAFs) of the identified significant risk factors will be calculated using the formula:

$$
\mathrm{PAF}=\frac{\sum_{\mathrm{i}=1}^{\mathrm{n}} \mathrm{P}_{\mathrm{i}} \mathrm{HR}_{\mathrm{i}}-\sum_{\mathrm{i}=1}^{\mathrm{n}} \mathrm{P}_{\mathrm{i}}^{\prime} \mathrm{HR}_{\mathrm{i}}}{\sum_{\mathrm{i}=1}^{\mathrm{n}} \mathrm{P}_{\mathrm{i}} \mathrm{HR}_{\mathrm{i}}}
$$

Where $\mathrm{Pi}=$ proportion of population at exposure level $\mathrm{i}$, current exposure 
- $\mathrm{P}^{\prime} \mathrm{i}=$ proportion of population at exposure level $\mathrm{i}$, counterfactual or ideal level of exposure

- $\mathrm{HR}=$ the relative risk at exposure level $\mathrm{i}$

- $\mathrm{n}=$ the number of exposure levels

Power estimation: Once the number of child and maternal deaths are calculated from the data, a post hoc power estimation will be performed to estimate the minimum effect size that will be detectable when identifying risk factors associated with each outcome with $80 \%$ power and $95 \%$ confidence. This will be calculated using PASS12 software. ${ }^{22}$

\section{Study outcomes}

The primary outcomes will be to establish if there are any hot spots relating to child $(<5)$ and maternal mortality in the study area, as this will indicate areas in which access to resources at community and individual levels needs to be reviewed.

Identifying a 'best model' to predict child and maternal mortality will enable other studies with similar data to be analysed to ensure health promotion. Understanding the relationship between maternal and child deaths will be important to improve service delivery in other rural areas, where the mother's health is compromised by HIV/AIDS, and access to reliable and affordable good quality healthcare and public health services is compromised.

\section{DISCUSSION}

South Africa has made slow progress towards achieving its MDGs 4 and 5, and failed to meet its 2015 targets, as did many other developing counties. ${ }^{1}$ This study will demonstrate the usefulness of spatiotemporal techniques in highlighting high-risk areas for future targeting of health interventions, as well as focusing more detailed research regarding the underlying risk factors (at the individual, household or community level) that may be driving these spatial-temporal mortality patterns. Many studies establishing the levels, trends and risk factors of child and maternal mortality have been carried out in rural South Africa, but few have fully documented the complex spatial-temporal patterns and risk factors using correlated mortality data.

This study will identify space-time clustering and develop a context-specific causal determinants framework of child and maternal mortality to guide statistical modelling approaches in a typical rural South African population. The findings from this multicomponent study will be used to develop a more refined intervention model for this rural South African population, which would have utility in other similar settings within the country, and potentially within the region. Knowledge of spatial patterns is essential to understanding the processes that link individual demographic outcomes to characteristics of a place, and to identifying mortality hot spots. This will assist the policymakers in planning and developing intervention measures to reduce child and maternal mortality in rural South Africa.

A limitation of the study is that it is not always easy to collect data in rural parts of developing countries, making it difficult to achieve a record of all deaths, which could have an impact on the results. Similarly, there are missed infant and child deaths due to mothers migrating out of the area after death but prior to the next census routine. However, the ACDIS specially trained tracking team attempts to find individuals in their new residences, which may be as far as Johannesburg or Durban.

Acknowledgements The authors thank Africa Centre for agreeing to provide us with the necessary data for this study. The authors acknowledge the contribution of Dickman Gareta, who will be extracting the required analytical data from the ACDIS for this study.

Contributors BT reviewed the literature, made substantial contributions to the conception and design and drafted the manuscript. BS participated in the design of the study and helped to draft the manuscript. FT participated in the design and coordination of the study, acquisition of data and helped to draft the manuscript. All authors read and approved the final manuscript.

\section{Competing interests None declared}

Ethics approval Biomedical Research Ethics Committee (BREC) at the University of KwaZulu-Natal (BE 169/15).

Provenance and peer review Not commissioned; externally peer reviewed.

Data sharing statement All authors read and approved the final manuscript.

Open Access This is an Open Access article distributed in accordance with the Creative Commons Attribution Non Commercial (CC BY-NC 4.0) license, which permits others to distribute, remix, adapt, build upon this work noncommercially, and license their derivative works on different terms, provided the original work is properly cited and the use is non-commercial. See: http:// creativecommons.org/licenses/by-nc/4.0/

\section{REFERENCES}

1. UN Inter-agency Group, Levels \& trends in child mortality. New York: WHO, 2015

2. Danzhen $\mathrm{Y}$, Philip B, Jingxian W, et al. Levels and trends in child mortality: estimates developed by the UN Inter-agency Group for child Mortality Estimation (IGME)-report 2013. Washington, DC, 2013.

3. Philadelphia P. South Africa reverses mortality trend in children under 5. The Global Sorce for Science News. 7 Oct 2013.

4. WHO. UNICEF, UNFPA, WORLD BANK GROUP and United Nations Population Division. Trends in maternal mortality: 1990 to 2015 Estimates by WHO, UNICEF, UNFPA, World Bank Group and the United Nations Population Division. Geneva: World Health Organization, 2015

5. WHO. Children: reducing mortality. World Health Organization, 2014.

6. UNAIDS. How AIDS changed everything - MDG6: 15 years, 15 lessons of hope from the AIDS response. 2015.

7. Razzaque A, Hossain AM, DaVanzo J, et al. Effect of Maternal Mortality on Survival of Under 5 Children. Asian Population Studies 2014;10:60-74.

8. Houle B, Clark SJ, Kahn K, et al. The impacts of maternal mortality and cause of death on children's risk of dying in rural South Africa: evidence from a population based surveillance study (1992-2013). Reprod Health 2015;12(Suppl 1):S7.

9. Burton R. Maternal health: there is cause for optimism. $S$ Afr Med J 2013;103:520-1.

10. Yego F, D'Este C, Byles J, et al. Risk factors for maternal mortality in a Tertiary Hospital in Kenya: a case control study. BMC Pregnancy Childbirth 2014;14.

11. Mosley HW, Chen LC. An analytical framework for the study of child survival in developing countries. Population and Development Review 1984;10(Suppl):25-45.

12. Meade JE. The Growing Economy by James E. Meade. Journal of Finance 1970;25:190-3. 
13. Houle B, Stein A, Kahn K, et al. Household context and child mortality in rural South Africa: the effects of birth spacing, shared mortality, household composition and socio-economic status. Int J Epidemiol 2013;10:1-11.

14. Tanser F, Hosegood V, Bärnighausen T, et al. Cohort Profile: Africa Centre Demographic Information System (ACDIS) and population-based HIV survey. Int J Epidemiol 2008;37:956-62.

15. WHO, UNICEF, UNFPA. The United Nations Population Division and The World Bank. The World Bank IBRD. IDA. 2015. http://data. worldbank.org/indicator/SH.STA.MMRT (accessed 12 Jan 2106).

16. Nabukalu D, Grobusch KK, Herbst K, Newell ML. Mortality in women of reproductive age in rural South Africa. Glob Health Action 2013;6:22834.
17. Anselin L, Bell DA. Spatial Fixed Effects and Spatial dependence. Tempe. 2011.

18. Gilks W, Richardson S, Spiegelhalter D. Markov chain mont carlo in practice. London: Chapman and Hall, 1996.

19. Gelman A, Rubin DB. Inference from iterative simulations using multiple sequences. Stat Sci 1992;7:457-72.

20. Spiegelhalter D, Best N, Carlin B, et al. Bayesian measures of model complexity and fit. J Roy Stat Soc 2002;64:583-639.

21. Sartorius B. Modelling determinants, impact, and spacetime risk of age-specific mortality in rural South Africa: integrating methods to enhance policy relevance. Global Health Action 2013;6:45.

22. Hintze J. PASS 12.NCSS,LLC. 2013. http://www.ncss.com (accessed 31 Jul 2015). 